\title{
The transition in the ventral stream from feature to real-world entity representations
}

\author{
Guy A. Orban ${ }^{1}$ *, Qi Zhu ${ }^{2}$ and Wim Vanduffel ${ }^{2}$ \\ ${ }^{1}$ Department of Neuroscience, University of Parma, Parma, Italy \\ 2 Laboratorium voor Neuro-en Psychofysiologie, Department of Neuroscience, KU Leuven, Leuven, Belgium
}

\section{Edited by:}

Chris Fields, New Mexico State

University, USA (Retired)

\section{Reviewed by:}

Natasha Sigala, University of Sussex UK

Shin'Ya Nishida, NTT Communication Science Laboratories, Japan

\section{*Correspondence:}

Guy A. Orban, Department of Neuroscience, University of Parma, Via Volturno 39, 43100 Parma, Italy e-mail:guy.orban@med.kuleuven.be
We propose that the ventral visual pathway of human and non-human primates is organized into three levels: (1) ventral retinotopic cortex including what is known as TEO in the monkey but corresponds to V4A and PITd/v, and the phPIT cluster in humans, (2) area TE in the monkey and its homolog LOC and neighboring fusiform regions, and more speculatively, (3) TGv in the monkey and its possible human equivalent, the temporal pole. We attribute to these levels the visual representations of features, partial real-world entities (RWEs), and known, complete RWEs, respectively. Furthermore, we propose that the middle level, TE and its homolog, is organized into three parallel substreams, lower bank STS, dorsal convexity of $\mathrm{TE}$, and ventral convexity of $\mathrm{TE}$, as are their corresponding human regions. These presumably process shape in depth, 2D shape and material properties, respectively, to construct RWE representations.

Keywords: 3D shape, retinotopy, actions, 2D shape, material properties

\section{INTRODUCTION}

This brief thought-provoking perspective paper complements the review devoted to the extrastriate neuronal properties published in Physiological reviews (Orban, 2008). At that time (Orban, 2008; Nassi and Callaway, 2009) the properties of infero-temporal neurons were not well understood, preventing a coherent picture of the function of monkey $\mathrm{TE}$ and its equivalent regions in man to be drawn. The present perspective paper attempts to correct

Abbreviations: Cortical areas and regions: AIP, anterior intraparietal area; CIP, caudal intraparietal area; DP, dorsal parietal area, located dorsal from V4; FST, fundus of superior temporal area, third element of the MT cluster; pFST, human homolog of FST; IPS0-5 is a set of successive retinotopic areas near the intraparietal sulcus (IPS) defined solely by reversal of polar angle (visual field is defined by both polar angle and eccentricity); IPS0-1 corresponds to V7/V7A; IT, infero-temporal cortex, includes three cytoarchitectonic fields, TEO, TE, and TGv; the first two have also been parceled into three antero-posterior subdivisions, posterior IT (PIT), central IT (CIT) and anterior IT (AIT), with PIT largely corresponding to TEO and CIT and AIT to TE; It includes the lower bank of the superior temporal sulcus (STS); LO1, LO2 lateral occipital area 1 and 2; LOC, lateral occipital cortex defined by the contrast intact vs scrambled images of objects. Includes LO1-2 but extends rostrally into occipito-temporal sulcus and fusiform cortex; LST, lateral superior temporal area, a motion area located in the monkey STS in front of FST; MSTv, medial superior temporal area ventral part, second component of the MT cluster; pMSTv human homologue of MSTv; MT, middle temporal area; first element of the MT cluster; OTd, occipito-temporal dorsal area; PFG, cytoarchitectonic field in IPL (others are PF, PG, and opt); PITd, posterior infero-temporal dorsal area; phPITd, putative human homologue of PITd; PITv, posterior infero-temporal ventral area; phPITv, putative human homologue of PITv; PPC, posterior parietal cortex (part of parietal cortex behind primary somato-sensory cortex); STPm, superior temporal posterior middle area, a motion area located in the upper bank of monkey STS (middle level); TF, TH cytoarchtectonic regions of parahippocampal cortex; TFO, cytoarchitectonic area posterior to TF/TH and medial to TEO; has been labeled previously VTF (visual part of TF) by Boussaoud et al. (1991), but is now recognized as a separate cytoarchitectonic entity ( Kravitz et al., 2013); V1,V2-V7, visual area 1, 2, to 7. The designation "V7" has been used only in humans; V5 corresponds to MT; While homology for V1-3 and V5/MT and V6 is relatively well established, hV4 refers a human area in positioned similarly to monkey $\mathrm{V} 4$ but having a different retinotopic organization; V3A, V4A, ad V7A, areas in neighborhood of V3, V4, and V7; V4t, fourth area of the MT cluster, initially considered incomplete now, accepted as corresponding to this shortcoming. Since fMRI became available (Dubowitz et al., 1998; Logothetis et al., 1998; Stefanacci et al., 1998; Vanduffel et al., 1998) for systematic investigation in the alert monkey (Vanduffel et al., 2001), considerable progress has been made, through fMRIguided monkey single-cell studies, and by parallel comparative imaging in humans and monkeys. In addition, the connections of TE cortex have recently been reassessed (Saleem et al., 2007, 2008; Ungerleider et al., 2008; Gerbella et al., 2010; Kravitz et al., 2013), allowing a tight comparison between anatomical connectivity and functionality.

\section{RETINOTOPIC ORGANIZATION OF THE VISUAL SYSTEM}

Our understanding of the retinotopic organization of the human visual system is largely due to fMRI. It is now established that human occipital cortex and neighboring parts of temporal and parietal cortex includes 15-17 distinct representations of the visual field. In addition to the three early visual areas V1-3, there is agreement (Wandell et al., 2007; Arcaro et al., 2009; Kolster et al., 2010) concerning hV4, LO1-2, the four areas of the MT cluster (MT, pMSTv, pFST, and pV4t), phPITd and phPITv (Figure 1A), and V6 (Pitzalis et al., 2006). There still is debate concerning the V3A complex which is subdivided into either two (V3A/B; Larsson and Heeger, 2006) or four areas (V3A/B/C/D; Georgieva et al., 2009). Dorsally, the V3A complex is bordered by V7 (Tootell etal., 1997), which is in fact the first parietal area, also designated IPS0 (Silver et al., 2005). Recently, V7 was reported to be part of a cluster of two areas, V7 (IPS0) and V7A

a complete hemifield; $\mathrm{pV} 4 \mathrm{t}$, human homologue of $\mathrm{V} 4 \mathrm{t} ; \mathrm{VO} 1, \mathrm{VO}$, ventral occipital area 1 and 2; Anatomical structures: IPS intraparietal sulcus separating the superior parietal lobule (SPL) from the inferior parietal lobule (IPL); MTG, middle temporal gyrus; OTS occipito-temporal sulcus; STS, superior temporal sulcus; STG, superior temporal gyrus; TPJ, temporo-parietal junction; Other abbreviations: AL, anterior lateral (face patch); BM, biological motion; ML, middle lateral (face patch); RWE, real world entity. 

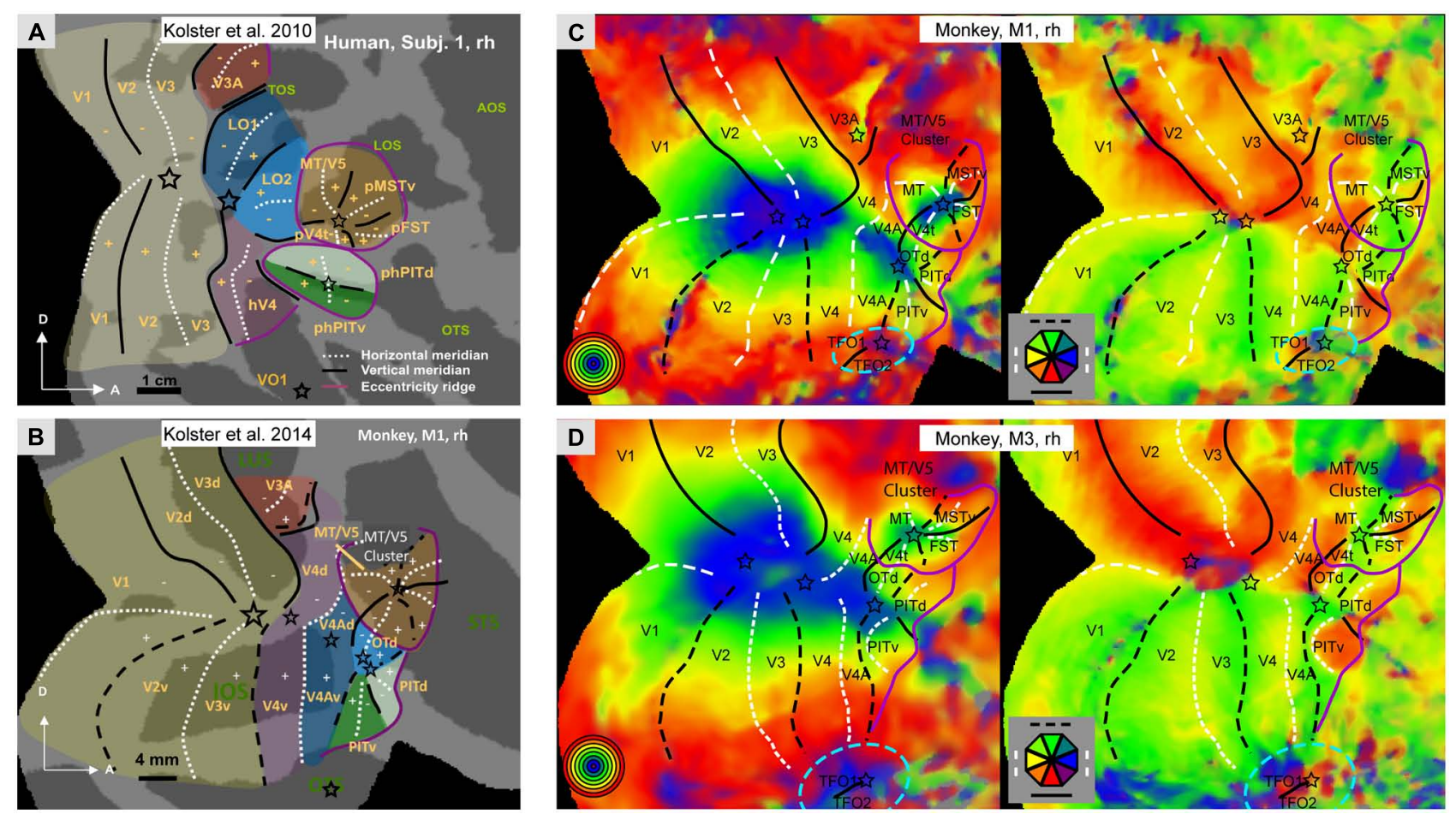

FIGURE 1 | (A,B) Schematic representation of the retinotopic organization of occipital cortex: in humans (A, subject 1, rh) and in monkeys (B, monkey M1, rh); Modified from Kolster etal. (2014).C,D: Polar angle and eccentricity maps for monkeys M1 (C) and M3 (D), same data as Janssens et al. (2014) but lower threshold. Black lines: vertical meridians (full: upper, dashed: lower), white dashed lines: horizontal meridians, stars: central visual field representation; purple lines: eccentricity ridges; In A,B: LuS: Iunate sulcus, STS: superior temporal sulcus; OTS occipito-temporal sulcus; TOS: transverse occipital sulcus, LOS: lateral occipital sulcus, AOS: anterior occipital sulcus, OTS occipito-temporal sulcus; Other nomenclature: see Abbreviations. In C,D blue stippled elliptic outlines mark additional retinotopic regions (TFO1/2) ventral to V4A/PITV.
(IPS1), sharing a central representation (Georgieva et al., 2009), a finding confirmed by using stereoscopically- instead of luminancedefined phase-encoded retinotopy stimuli (Kolster et al., 2011). This test also suggested that at more rostral levels the posterior parietal cortex (PPC) is retinotopically organized into 3-6 additional areas. Their complete characterization requires further work, since investigations thus far have relied mainly on polar angle analyses to define IPS2-5 (Silver and Kastner, 2009). On the other, ventral side of the occipital cortex Kolster et al. (2010) have described a single VO1 area (Figure 1A), although these data are also compatible with the presence of a second VO2 area, as described by Brewer et al. (2005). Finally, Arcaro et al. (2009) have shown that VO1-2 borders two additional retinotopic areas, $\mathrm{PH} 1$ and $\mathrm{PH} 2$, extending into the parahippocampal cortex. Thus in humans, a major difference exists between the dorsal and ventral visual pathways with respect to their retinotopic representation. The dorsal pathway retains a retinotopic organization, while the ventral pathway discards this organization beyond the phPIT cluster. It needs to be noted, however, that the most ventrally located occipito-temporal cortex processing scene information remains retinotopically organized. It has been suggested that at higher levels of the ventral pathway, eccentricity remains an important principle of organization (Levy et al., 2001), but this largely reflects the representation of large eccentricities in scene-processing regions.
The situation is very similar in the macaque. Its occipital cortex and neighboring parts of temporal and parietal cortex includes 14 retinotopic maps (Figure 1B): the three early areas V1-3, V4, and its two satellites (V4A and OTd), the two PITs (Janssens et al., 2014; Kolster etal., 2014), V3A, the four areas of the MT cluster (Kolster et al., 2009), and V6 in the parieto-occipital sulcus (Galletti et al., 1999). Cytoarchitectonic area TEO, which initially was proposed to contain a single retinotopic map (Boussaoud et al., 1991), in fact includes four different retinotopic maps: V4A, OTd, PITd, and PITv (Janssens et al., 2014; Kolster et al., 2014). It may be that neighboring cytoarchitectonic area TFO will undergo the same fate. Indeed, ventrally in occipital cortex, in front of the most peripheral part of V4 and below V4A, there is preliminary evidence (Janssens et al., 2014; Kolster et al., 2014) for another central representation, defining a cluster including two areas joined by that central representation. These areas have been tentatively labeled TFO1 and TFO2 (Figures 1C,D). The location in the dorsal bank of OTS and internal organization of this cluster suggest they may correspond to VO1-2 of humans. In humans VO1/2 are sensitive to color (Brewer et al., 2005) and color responses have been reported in a monkey PET study in a region that likely corresponds to TFO (Takechi et al., 1997). We propose that TFO $1 / 2$ are the starting point of the scene-processing pathway, consistent with recent fMRI activation and single cell recordings (Kornblith et al., 2013, but see Nasr et al., 2011). As 
in humans this pathway emphasizes the peripheral visual field (Kravitz etal., 2013). A number of parietal regions are retinotopically organized. Arcaro et al. (2011) described, in addition to DP, a pair of areas, CIP1 and CIP2, in the caudal part of the lateral bank of the IPS. In keeping with their location caudal to an extensive representation of peripheral visual field, CIP1/2 might be the monkey counterparts of the V7/V7A pair (Durand et al., 2009). This implies that human areas V3B-D have no counterpart in the monkey and are evolutionary novel areas. This is consistent with the caudal elongation of the IPS which in humans includes an occipital portion needed to bridge the enlargement of IPL (Grefkes and Fink, 2005). Further forward in monkey IPS, Arcaro et al. (2011) described a single hemifield representation, LIP, of which the central representation had been described by Fize et al. (2003).

In summary, the retinotopic organization of occipital cortex is remarkably similar in human and non-human primates, more than initially appreciated (Wandell et al., 2007). In addition, the organization beyond occipital cortex is also rather similar. The dorsal visual pathway of both humans and monkeys maintains a retinotopic organization, while the ventral pathway abandons this organization beyond TEO/the PIT monkey areas and their human homologs (phPITs). In both species the rostral limit of retinotopic cortex represents the peripheral visual field (purple lines in Figure 1). The most ventral, scene-processing pathway transiting through the parahippocampal cortex retains this organization at least in humans and possibly in monkeys (this ventral cortex is difficult to image in the monkey given the susceptibility artifacts, see Ku et al., 2011). Insofar as scene processing might be considered the qualitative counterpart of the metric processing of space in the dorsal pathway, the underlying principle may be that areas processing space, either quantitatively or qualitatively retain a crude retinotopic organization. In the monkey, the temporal cortex beyond TEO/the PITs includes mainly areas TE and TGv near the temporal pole (Figure 2A). In humans, LOC, which primarily corresponds to TE (Denys et al., 2004; Sawamura et al., 2005) is located several $\mathrm{cm}$ away from the temporal pole, suggesting that the TGv region has greatly expanded in humans. This raises the question by which functional organization principle, if any, the retinotopic organization has been replaced in these regions of temporal cortex.

\section{PITd PROCESSES 3D SHAPE FROM SHADING, ONE OF THE BUILDING BLOCKS OF SHAPE REPRESENTATION FOR REAL-WORLD ENTITIES}

In monkeys, the fMRI study of Nelissen et al. (2009) indicates that the dorsal PIT is involved in processing 3D shape from shading. The fMRI activation of PITd corresponds to stronger neuronal responses for shading patterns reflecting 3D structure (Köteles et al., 2008). In humans, 3D shape from shading is similarly processed in a restricted occipito-temporal region (Georgieva etal., 2008). Matching the local maximum of this activation to a maximum-probability map of occipital retinotopic areas (Abdollahi et al., 2013) suggests that it is located near or in phPITd. In an effort to dissociate 3D shape from shading from simple flat luminance patterns, both Nelissen et al. (2009) and Georgieva et al. (2008) required joined activation in several specific contrasts for a region to be considered processing 3D shape from shading. Sereno et al. (2002) also reported 3D shape from shading responses in a somewhat broader region near PITd, including MT and FST in which several 3D shape cues, motion, shading, and texture converged. The importance of these observations derives from the fact that the image of any real-world object is necessarily (because of optics) characterized by two complementary components: a boundary that defines its $2 \mathrm{D}$ shape and a luminance pattern inside this boundary that defines its relief (shape in depth or 3D shape). These two complementary components depend in complex ways on the material properties and shape of the objects, as well as the direct and indirect light sources present in the scene. Nevertheless, 2D shape and 3D shape from shading combine to unambiguously define a visual representation of a real-world entity (RWE), whether an object, a plant, an animal, or a conspecific. RWE is preferred to the term object which is ambiguous, as the above listing shows. It is well established that boundary information is processed in V4 (Pasupathy and Connor, 2001) and is further elaborated in what is commonly called TEO (Brincat and Connor, 2004). Thus the most rostral retinotopic regions of the ventral pathway (Figure 1B), parts of cytoarchitectonic TEO, contain the elements required to generate visual representations of RWE. We propose that the primary function of TE, located beyond the retinotopic cortex, is to house the visual representations of RWEs, built by combining lowerlevel inputs from retinotopic cortex. The visual representation of RWEs can also be triggered by their images (Tanaka et al., 1991), and by even more simplified stimuli such as drawings (Denys et al., 2004).

The visual representations of RWEs are supposedly assembled in TE by combining inputs representing a boundary (or external contour) as well as elements of the luminance distribution inside that boundary. These internal elements can be either contours corresponding to extremes in the luminance distribution, or regions of constant or smoothly varying luminance. Indeed, this combinatorial view is supported by recent recordings in the ML face patch of the monkey, located just at the edge of retinotopic cortex. Almost all neurons in this patch are face selective (Tsao et al., 2006) and this selectivity arises from combining the geometry of the boundary with that of key internal features such as the eyes, nose, or mouth (Freiwald et al., 2009), but also includes the contrast levels in certain positions with respect to these features (Ohayon et al., 2012). However, this combination of 2D shape and 3D from shading does not exhaust the possible visual representations of RWEs, since the nature of RWEs is specified by not only their shape but also their material properties. Hence the representation of RWEs is build up from three main sources: features related to the $2 \mathrm{D}$ shape of the boundary in the image, and to the 3D shape, and material properties of the region enclosed by the boundary.

\section{REPRESENTATIONS OF REAL-WORLD ENTITIES IN TE}

Recent anatomical data suggest that three parallel substreams operate within TE (Figure 2A), located in the lower bank of STS and in the dorsal and ventral parts of TE. We suggest that these three streams preferentially use features of $3 \mathrm{D}$ shape, $2 \mathrm{D}$ shape, and material properties, respectively, to build up RWE 


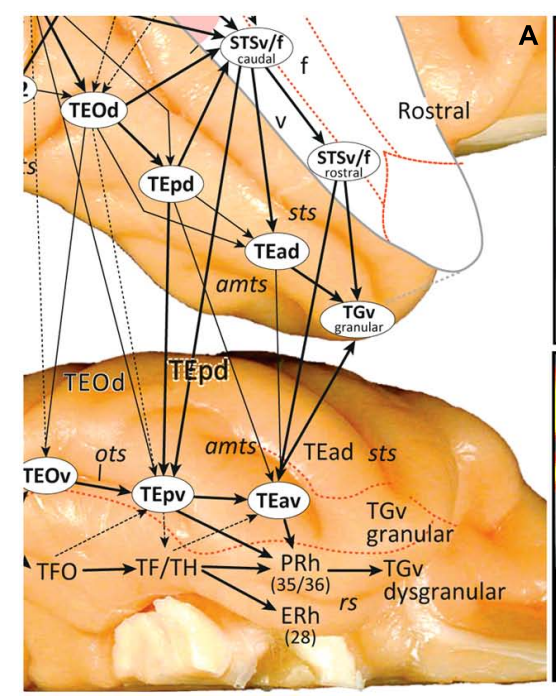

FIGURE 2 | (A) The anatomical organization of monkey TE into three parallel substreams (from Kravitz et al., 2013); (B-E) SPMs showing activation sites in right IT for 2D shape, color, shape vs. no shape, and gloss. These were defined by the following subtractions: intact vs. scrambled images of objects (B), color vs. no color mondrians (C), inact vs. scrambled images of objects
(D) main effect of gloss, independent of contrast (E). In D the non-shape, selective voxels were strongly selective for material property, whereas shape-selective ones were not. Purple curved lines in B-E: approximate caudal boundary of TE. From Denys et al. (2004; B), Harada et al. (2009; C), Goda etal. (2014; D), and Okazawa etal. (2012; E). representations (Figure 3). This implies that functional segregation between these substreams is maximal at the transition between the retinotopic, feature level and the middle level (i.e., the TEO/TEp border in Figures 2A,D) and gradually blurs toward the rostral end of TE. Indeed, the three aspects defining RWEs (3D shape, $2 \mathrm{D}$ shape, and material properties) contribute in different proportions to the definition of given RWEs, and some cues belonging to one of the aspects may remain represented at more rostral levels, as for example color, one of the material cues (see below). According to this scheme the middle substream carries mainly 2D shape information, as evidenced by the subtraction

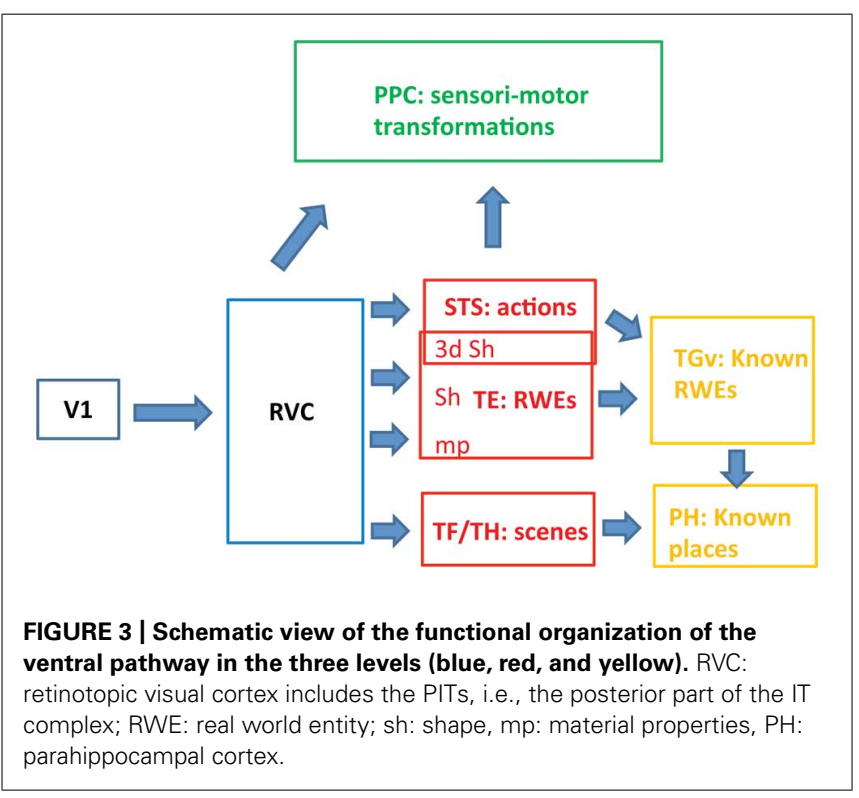

intact minus scrambled images of objects, which mainly activates dorsal TE (Figure 2B; Denys et al., 2004; Sawamura et al., 2005; Lafer-Sousa and Conway, 2013). A long list of single-cell studies have been devoted to 2D shape selectivity in IT cortex (Logothetis and Sheinberg, 1996; Tanaka, 1996; Orban, 2008 for review), with some stressing the affine nature of the representation (Kayaert et al., 2005). This 2D shape substream also contains several face patches, such as the ML, and AL patches (Moeller et al., 2008).

The ventral TE substream may process material properties (for review see Fleming, 2014) which also contribute to the definition of RWEs (e.g., a tomato is red and smooth). This is supported by the color activation sites in ventral TE (Figure 2C; Harada et al., 2009; Lafer-Sousa and Conway, 2013). The other principal material property cue is texture (texture is also a cue for $3 \mathrm{D}$ shape; see Sereno et al., 2002; Orban, 2011). Little is known about texture processing in monkeys (see Köteles et al., 2008), but in humans ventral occipito-temporal cortex is heavily involved in texture processing (Peuskens et al., 2004; Cant and Goodale, 2007). Ku et al. (2011) have reported face patches in and around the ventral temporal cortex of the monkey: in ventral TE, area TF, entorhinal cortex, hippocampus, and region labeled ventral V4, which might have included TFO. Since the hairy monkey face and control stimuli (fruits, houses, and fractals) differed in texture, some of these activation sites (in particular the posterior ones) might actually reflect the texture differences rather than the presence of the face. Regions in PIT processing material properties have been investigated recently by Goda et al. (2014), who showed a clear segregation between shape and material properties at the level of PIT (Figure 2D), in agreement with our proposal. We propose that the third substream in the lower bank of STS processes 3D shape (Sereno et al., 2002; Yamane et al., 2008). This 
proposal is consistent with the presence in the lower bank of a small patch concerned with gloss (Figure 2E; Okazawa et al., 2012), a marker of 3D convexity for certain materials, and TEs, a region extracting curvature from disparity (Janssen et al., 2000). This substream overlaps with action-processing regions located in both banks of the STS, especially their deeper regions (Nelissen et al., 2011). One of the main cues for extracting actions is the deformation of body shape (Vangeneugden et al., 2009; Singer and Sheinberg, 2010), explaining the proximity of shape, and action processing areas. Similarly, material properties contribute heavily to scene processing, which may explain their location in ventral TE, as it neighbors the scene-processing stream in parahippocampal $\mathrm{TF} / \mathrm{TH}$

Both the general anatomy, that indicates serial processing (Figure 2A), and studies specific to the face-processing system suggest that the representation of RWEs might be further elaborated rostrally within TE. A detailed study of the face patches (Freiwald and Tsao, 2010) suggests that the first step is the extraction of the face category in ML; that additional properties, such as the viewpoint from which the face is seen, are represented in subsequent patches; and that finally at the highest level, exemplars, individual faces, are represented, implying that sufficient invariance has been achieved. Similarly Lafer-Sousa and Conway (2013) have suggested that the representation of color is more elaborated in anterior than in posterior TE. Koida and Komatsu (2007) demonstrated the task dependent activity of TE color selective neurons. Task dependent processing and other aspects of TE processing such as extending the neural representation beyond the stimulus presentation (Kovacs et al., 1995) or buffering the last representation (Orban and Vogels, 1998) are beyond the scope of the present perspective paper.

Despite this elaboration of RWE representations, including becoming gradually more invariant (DiCarlo et al., 2012), the representation in TE remains incomplete in the sense that the entire RWE is generally not represented (a few neurons may do so, as suggested for target-paired association neurons; Hirabayashi et al., 2013). Even in the anterior face patches, only the face is represented, not the whole person; also, patches related to color represent only one material aspect of the RWE. The partial representation of the RWE at the middle level can be considered a generalization of the selectivity of TE neurons for 2D shape components (Tanaka et al., 1991). The RFs of TE neurons are relatively large (about $15^{\circ}$ diameter), located primarily in the contralateral visual field, and generally included the fovea (Op De Beeck and Vogels, 2000). Hence a certain spatial coding remains possible, in particular that of the relative positions of shape or RWE parts. Several rationales can be advanced for the incomplete representation of RWEs in TE having to do with more flexible representations. In particular, some material properties define the exemplar but not the category (e.g., John may have black hair but not all men have black hair), accommodation of slow changes in properties, e.g., due to aging, or seasons (color changes of the leaves), and finally detection of uncommon associations of shape and color (see Zeki and Marini, 1998; e.g., John generally looks healthy, but can be very pale because of illness).

Thus far, views about the organization of TE have been dominated by the presence of patches in TE, among which face and body patches (Tsao et al., 2003; Pinsk et al., 2009; Bell et al., 2011; Popivanov et al., 2012) are the best known. Initially it was assumed that the non-face and non-body objects were processed outside these patches (Ishai et al., 1999; Tsao et al., 2003), implying that RWE of different types were processed in different compartments of TE. This view, however, is inconsistent with recent evidence for patches for color, 3D shape from disparity, or gloss (Harada et al., 2009; Joly et al., 2009; Okazawa et al., 2012). A recent study by Srihasam et al. (2012) sheds new light on the exact organization of TE. These authors showed that when monkeys are trained to use numerical or letter symbols from a young age, these stimuli are represented in patches within TE, but are not present in untrained monkeys or those trained to use these symbols as adults (and not learning the task as well). While others (Vogels and Orban, 1994; Kobatake et al., 1998; Sigala and Logothetis, 2002) have reported plasticity at the single-cell level after training, the Srihasam study was the first to report functional architectural changes in TE, rather than just changes in neuronal properties. Srihasam et al. (2012) suggest that patches arise because neurons with similar selectivity tend to group together to increase computational efficiency (shorter connections). In retinotopic cortex, these groupings are constrained by the retinotopic organization, but in TE this is not the case, thus giving rise to varying degrees of aggregation, probably depending on the behavioral relevance of the selectivity. Those aspects or components of RWEs with strong behavioral relevance are grouped into complex systems of multiple connected patches, of which the face patches are probably the most elaborated. Those with limited relevance, such as properties/parts of objects encountered only infrequently, have small representations in columnar-like structures (Tanaka et al., 1991). Those with intermediate relevance have a somewhat broader representation, in one or two patches, such as color or 3D shape. Thus the processing of RWEs of different type or nature is interwoven, their properties being represented more or less extensively depending on behavioral relevance. Such size differences of functional TE modules are consistent with the findings of Sato et al. (2013), with our largest and smallest modules corresponding to their domains and columns, respectively. In humans these domains may include the word form areas (Cohen et al., 2000) analyzing strings of symbols during reading, even if words are not actually RWEs.

\section{REPRESENTATIONS OF ACTIONS IN STS}

Several lines of investigation suggest that actions (purposeful movements of an agent: animal, human, or even robot) are processed in the middle and rostral STS largely in parallel with RWEs in TE (Figure 3). Recent evidence suggests that actions are extracted in LST and STPm, two motion-sensitive regions just anterior to the MT cluster. In these regions the configuration and kinematic cues of BM interact (Jastorff et al., 2012), which is the definition of action. Indeed, action-selective neurons have been recorded at this level, and both cues appear operative: deforming shape in the lower bank, and motion patterns in the upper bank (Vangeneugden et al., 2009). We have begun to understand the homology of monkey STS (Orban and Jastorff, 2014): The lower bank corresponds to posterior OTS and fusiform cortex in humans, overlapping with LOC (in which actions and shape 
overlap, as in the lower bank of STS; Jastorff and Orban, 2009), while the upper bank of monkey STS corresponds to posterior MTG and posterior STS in humans (Jastorff and Orban, 2009; Jastorff et al., 2012).

We have recently shown that the action-sensitive regions of STS devoted to grasping project to the ventral premotor cortex (F5), where mirror neurons occur, via two way stations in the PPC: AIP and PFG (Nelissen et al., 2011). We believe that this is a general strategy within the primate visual system, not merely for grasping and manipulative actions, but for all types of action. The STS action-processing regions project to the PPC in order to extract action category which requires that a large number of invariances to be solved: not only for size, position, and in plane orientation, as for RWEs, but also for viewpoint and posture. The available evidence (Freiwald and Tsao, 2010) suggests that TE and neighboring regions achieve invariance only at the expense of large neuronal pools and that therefore the many invariances required for understanding body actions involve too much neuronal hardware to be realistically achieved in the STS. Hence, we propose that the STS regions send the visual information about which action is observed to the PPC housing the schema of specific actions, i.e., the sensori-motor transformation underlying various actions. By projecting these visual signals onto the corresponding motor plan, invariance is automatically achieved and categorization becomes feasible. This invariance problem is less stringent for facial expressions, as the viewpoints, and postural invariance requirements are much more limited. Hence what applies to body action may not necessarily apply to facial expressions, explaining the presence of face patches in the upper bank of STS, where dynamic face expressions are processed (Polosecki et al., 2013).

These action signals sent to the PPC concern the nature/goal of the action defining which action is observed. However, actions are also further processed in the STS itself, analysis probably related to how the action is performed, e.g., slowly or quickly, with difficulty or easily, physiologically or pathologically. The latter sort of processing provides information about the state of the actor, even if the actor itself, an RWE, is processed in TE. The state of the agent reflects his/her emotions, but also the physiological state, and perhaps also vitality (Di Cesare et al., 2013). The latter aspect is related to the rank of the actor in the group or the social organization in general and may be dealt with in human TPJ, a region which may have arisen from some middle part of the STS (Sallet et al., 2011; Mars et al., 2013). TPJ is often considered the starting point (Saxe et al., 2004) for processing other agents (theory of mind), but recent studies (Jastorff and Orban, 2009) alternatively suggest that there might be a representation of an agent in the scene in posterior STG. Activity in posterior STS and TPJ would then specify properties of the agent, such as rational or efficient behavior (Jastorff et al., 2011).

\section{THREE LEVELS OF PROCESSING IN THE VENTRAL STREAM (FIGURE 3)}

TE corresponds to the middle level of the ventral stream in the monkey. It builds a partial representation of RWEs and operates in parallel with STS, processing actions and TF/TH processing scenes (Figure 3). TE receives input from retinotopic cortex (first level) where image features are processed to generate higher-order features related to 3D shape, 2D shape, or material properties in specific parts of the visual field. The retinotopic visual cortex not only processes a range of elementary image features (Zeki, 1978) but also resolves image segmentation by establishing topological relationships between the features: inside vs. outside and in front vs. behind (Zhang and von der Heydt, 2010). The anatomy indicates, however, that the ventral pathway in monkeys may include, in addition to the retinotopic cortex and TE, a third level beyond TE. A small temporal region, TGv, receiving input from the three substreams in TE, is situated in front of TE near the temporal pole (Kravitz et al., 2013). The TGv region projects to rhinal cortex in which memory of the association between two images is constructed by the convergence of their representations in TE (Naya et al., 2003a; Hirabayashi et al., 2013). We propose that the TGv region, which is greatly expanded in humans and is referred to as the temporal pole, builds on the partial representations of individual RWEs achieved at the rostral TE (Freiwald and Tsao, 2010) to generate representations of known RWEs (Damasio et al., 2004; Quiroga et al., 2005). The association of the elements present in TE detected in rhinal cortex (Hirabayashi et al., 2013), may be backprojected (Naya et al., 2003b; Takeuchi et al., 2011) onto the most rostral visual part of temporal cortex, giving rise to representations of known RWEs (Takeda et al., 2005). For example, exemplars of a shape category, e.g., face plus body, and particular material properties define a given individual and this association gives rise to the representation of that known individual in TGv, perhaps supplemented by information about how he acts and the scenes in which he appears. In contrast to the TE level, the representation here is that of the complete RWE, e.g., a conspecific, and no longer simply a face. A similar operation may be applied to scene information in parahippocampal areas, giving rise to known places, although no direct link between TF/TH and TGv has been described. Interestingly, recent fMRI data (Miyamoto etal., 2014) indicate that monkey rhinal cortex encodes familiar items, operationalized as middle items in a serial probe task. This type of encoding is appropriate for known RWEs, and by extension, semantic knowledge. In humans, this third level of the ventral stream, the temporal pole, may correspond to the anterior part of the semantic system (Vandenberghe et al., 1996). This association between the temporal pole and semantic memory has its basis in the connections of the pole to memory structures such as rhinal cortex. The third level may also be linked with the amygdala, the structure underlying association between known person and emotions, which has been referred to as personal semantic memory (Olson et al., 2007).

The visual representation of known RWEs at the third level also seems consistent with single cell recorded in the human hippocampal complex showing neuronal selectivity for familiar persons or places, sometimes referred to as visual concept neurons (Quiroga, 2012). This might suggest that visual episodes (events) are also represented at this third level and probably beyond, e.g., in entorhinal cortex and hippocampus. The latter view is supported by the recent study of Miyamoto et al. (2014), who showed that the memory trace of recalled items, operationalized as the first item in a serial probe task, is located in caudal entorhinal cortex and hippocampus of the monkey. A relatively small region may suffice for representing episodes, as this representation may be short-lived. Indeed, if the event is repeated or memorable it may become knowledge 
(the fact that somebody looks ill may become part of medicine or history); if it is important for the subject it may become part of autobiographic memory. The dissociation of episodic and semantic memory within the third, known-RWE level is also supported by patients studies (Hirni et al., 2013).

For simplicity we have described the three levels, those processing features, partial RWEs, and known RWEs, as separate components, using anatomy (Kravitz et al., 2013) as a guide. It is possible, however, that the transitions between these levels are gradual. Indeed, as mentioned, the ML face patch is located at the edge of retinotopic cortex and the overlap between retinotopic cortex and some of the more caudal face or body patches may be larger in humans than in monkeys. In the monkey, the body patch is anterior to the MT cluster (Jastorff et al., 2012), but in humans EBA overlaps the retinotopic MT cluster to a large extent (Ferri et al., 2012). Moreover, segregation between the third level, TGv, and the levels below, TE, and beyond, rhinal cortex, might be incomplete, insofar as the anterior parts of TE and the lower bank of STS also exchange bidirectional projections with rhinal cortex. At this level, differences between humans and monkeys may have arisen due to the enlargement of the temporal pole in humans.

The three levels of the ventral stream also appear to differ in the way they develop. The experiment of Srihasam et al. (2012) suggests that the middle level (TE, and by extension perhaps also STS and TF/TH) reflects the individual development, while the earlier retinotopic level is probably species-specific. This explains that although the different retinotopic regions are present in all individual subjects, albeit with some variation in size and location, the number of patches in TE seems more variable among individuals (Bell et al., 2011; Lafer-Sousa and Conway, 2013). The third and final level would remain the most plastic and dependent on lifelong mental activity. Its internal organization is presently unknown.

In conclusion we propose that the ventral stream is organized into three levels comprising the ventral retinotopic cortex known as TEO, TE, and TGv in the monkey, and their homologs in human cortex. We attribute to these levels the visual representation of features, partial RWEs, and more speculatively, known, complete RWEs, respectively. Furthermore, the middle level TE and its human equivalent is organized into three parallel substreams related to processing shape in depth, 2D shape, and material properties in order to build up RWE representations.

\section{ACKNOWLEDGMENTS}

This work was supported by ERC grant Parietalaction and IUAP grant 7/11.

\section{REFERENCES}

Abdollahi, R. O., Glasser, M. F., Dierker, D., VanEssen, D. C., and Orban, G. A. (2013). A probabilistic atlas of 18 human retinotopic areas. Soc. Neurosci. Abstr. $824: 15$.

Arcaro, M. J., McMains, S. A., Singer, B. D., and Kastner, S. (2009). Retinotopic organization of human ventral visual cortex. J. Neurosci. 29, 10638-10652. doi: 10.1523/JNEUROSCI.2807-09.2009

Arcaro, M. J., Pinsk, M. A., Li, X., and Kastner, S. (2011). Visuotopic organization of macaque posterior parietal cortex: a functional magnetic resonance imaging study. J. Neurosci. 31, 2064-2078. doi: 10.1523/JNEUROSCI.3334-10.2011

Bell, A. H., Malecek, N. J., Morin, E. L., Hadj-Bouziane, F., Tootell, R. B., and Ungerleider, L. G. (2011). Relationship between functional magnetic resonance imaging-identified regions and neuronal category selectivity. J. Neurosci. 31, 12229-12240. doi: 10.1523/JNEUROSCI.5865-10.2011

Boussaoud, D., Desimone, R., and Ungerleider, L. G. (1991). Visual topography of area TEO in the macaque. J. Comp. Neurol. 306, 554-575. doi: $10.1002 /$ cne. 903060403

Brewer, A. A., Liu, J., Wade, A. R., and Wandell, B. A. (2005). Visual field maps and stimulus selectivity in human ventral occipital cortex. Nat. Neurosci. 8, 11021109. doi: 10.1038/nn1507

Brincat, S. L., and Connor, C. E. (2004). Underlying principles of visual shape selectivity in posterior inferotemporal cortex. Nat. Neurosci. 7, 880-886. doi: $10.1038 / \mathrm{nn} 1278$

Cant, J. S., and Goodale, M. A. (2007). Attention to form or surface properties modulates different regions of human occipitotemporal cortex. Cereb. Cortex 17, 713-731. doi: 10.1093/cercor/bhk022

Cohen, L., Dehaene, S., Naccache, L., Lehéricy, S., Dehaene-Lambertz, G., Hénaff, M. A., et al. (2000). The visual word form area: spatial and temporal characterization of an initial stage of reading in normal subjects and posterior split-brain patients. Brain 123, 291-307. doi: 10.1093/brain/123.2.291

Damasio, H., Tranel, D., Grabowski, T., Adolphs, R., and Damasio, A. (2004). Neural systems behind word and concept retrieval. Cognition 92, 179-229. doi: 10.1016/j.cognition.2002.07.001

Denys, K., Vanduffel, W., Fize, D., Nelissen, K., Peuskens, H., Van Essen, D., et al. (2004). The processing of visual shape in the cerebral cortex of human and nonhuman primates: a functional magnetic resonance imaging study. J. Neurosci. 24, 2551-2565. doi: 10.1523/JNEUROSCI.3569-03.2004

Di Cesare, G., Di Dio, C., Rochat, M. J., Sinigaglia, C., Bruschweiler-Stern, N., Stern, D. N., et al. (2013). The neural correlates of 'vitality form' recognition: an fMRI study: this work is dedicated to Daniel Stern, whose immeasurable contribution to science has inspired our research. Soc. Cogn. Affect. Neurosci. doi: 10.1093/scan/nst068 [Epub ahead of print].

DiCarlo, J. J., Zoccolan, D., and Rust, N. C. (2012). How does the brain solve visual object recognition? Neuron 73, 415-434. doi: 10.1016/j.neuron.2012. 01.010

Dubowitz, D. J., Chen, D. Y., Atkinson, D. J., Grieve, K. L., Gillikin, B., Bradley, W. G., et al. (1998). Functional magnetic resonance imaging in macaque cortex. Neuroreport 9, 2213-2218. doi: 10.1097/00001756-199807130-00012

Durand, J. B., Peeters, R., Norman, J. F., Todd, J. T., and Orban, G. A. (2009). Parietal regions processing visual 3D shape extracted from disparity. Neuroimage 46, 1114-1126. doi: 10.1016/j.neuroimage.2009.03.023

Ferri, S., Kolster, H., Jastorff, J., and Orban, G. A. (2012). The overlap of the EBA and the MT/V5 cluster. Neuroimage 66C, 412-425. doi: 10.1016/j.neuroimage.2012.10.060

Fize, D., Vanduffel, W., Nelissen, K., Denys, K., Chef d'Hotel, C., Faugeras, O., et al. (2003). The retinotopic organization of primate dorsal V4 and surrounding areas: a functional magnetic resonance imaging study in awake monkeys. J. Neurosci. 23, 7395-7406.

Fleming, R. W. (2014). Visual perception of materials and their properties. Vision Res. 94, 62-75. doi: 10.1016/j.visres.2013.11.004

Freiwald, W. A., and Tsao, D. Y. (2010). Functional compartmentalization and viewpoint generalization within the macaque face-processing system. Science 330 , 845-851. doi: 10.1126/science. 1194908

Freiwald, W. A., Tsao, D. Y., and Livingstone, M. S. (2009). A face feature space in the macaque temporal lobe. Nat. Neurosci. 12, 1187-1196. doi: 10.1038/nn.2363

Galletti, C., Fattori, P., Gamberini, M., and Kutz, D. F. (1999). The cortical visual area V6: brain location and visual topography. Eur. J. Neurosci. 11, 3922-3936. doi: 10.1046/j.1460-9568.1999.00817.x

Georgieva, S., Peeters, R., Kolster, H., Todd, J. T., and Orban, G. A. (2009). The processing of three-dimensional shape from disparity in the human brain. $J$. Neurosci. 29, 727-742. doi: 10.1523/JNEUROSCI.4753-08.2009

Georgieva, S. S., Todd, J. T., Peeters, R., and Orban, G. A. (2008). The extraction of $3 \mathrm{D}$ shape from texture and shading in the human brain. Cereb. Cortex 18, 2416-2438. doi: 10.1093/cercor/bhn002

Gerbella, M., Belmalih, A., Borra, E., Rozzi, S., and Luppino, G. (2010). Cortical connections of the macaque caudal ventrolateral prefrontal areas $45 \mathrm{~A}$ and $45 \mathrm{~B}$. Cereb. Cortex 20, 141-168. doi: 10.1093/cercor/bhp087

Goda, N., Tachibana, A., Okazawa, G., and Komatsu, H. (2014). Representation of material properties of objects in the visual cortex of nonhuman primates. J. Neurosci. 34, 2660-2673. doi: 10.1523/JNEUROSCI.2593-13.2014 
Grefkes, C., and Fink, G. R. (2005). The functional organization of the intraparietal sulcus in humans and monkeys. J. Anat. 207, 3-17. doi: 10.1111/j.1469. 7580.2005.00426.x

Harada, T., Goda, N., Ogawa, T., Ito, M., Toyoda, H., Sadato, N., et al. (2009). Distribution of colour-selective activity in the monkey inferior temporal cortex revealed by functional magnetic resonance imaging. Eur. J. Neurosci. 30, 1960 1970. doi: 10.1111/j.1460-9568.2009.06995.x

Hirabayashi, T., Takeuchi, D., Tamura, K., and Miyashita, Y. (2013). Microcircuits for hierarchical elaboration of object coding across primate temporal areas. Science 341, 191-195. doi: 10.1126/science.1236927

Hirni, D. I., Kivisaari, S. L., Monsch, A. U., and Taylor, K. I. (2013). Distinct neuroanatomical bases of episodic and semantic memory performance in Alzheimer disease. Neuropsychologia 51, 930-937. doi: 10.1016/j.neuropsychologia.2013.01.013

Ishai, A., Ungerleider, L. G., Martin, A., Schouten, J. L., and Haxby, J. V. (1999). Distributed representation of objects in the human ventral visual pathway. Proc. Natl. Acad. Sci. U.S.A. 96, 9379-9384. doi: 10.1073/pnas.96.16.9379

Janssen, P., Vogels, R., and Orban, G. A. (2000). Selectivity for 3D shape that reveals distinct areas within macaque inferior temporal cortex. Science 288, 2054-2056. doi: 10.1126/science.288.5473.2054

Janssens, T., Zhu, Q., Popivanov, I. D., and Vanduffel, W. (2014). Probalistic and single-subject retinotopic maps reveal the topographic organization of face patches in the macaque cortex. J. Neurosci. (in press).

Jastorff, J., Clavagnier, S., Gergely, G., and Orban, G. A. (2011). Neural mechanisms of understanding rational actions: middle temporal gyrus activation by contextual violation. Cereb. Cortex 21, 318-329. doi: 10.1093/cercor/bhq098

Jastorff, J., and Orban, G. A. (2009). Human functional magnetic resonance imaging reveals separation and integration of shape and motion cues in biological motion processing. J. Neurosci. 29, 7315-7329. doi: 10.1523/JNEUROSCI.4870-08.2009

Jastorff, J., Popivanov, I. D., Vogels, R., Vanduffel, W., and Orban, G. A. (2012). Integration of shape and motion cues in biological motion processing in the monkey STS. Neuroimage 60, 911-921. doi: 10.1016/j.neuroimage.2011.12.087

Joly, O., Vanduffel, W., and Orban, G. A. (2009). The monkey ventral premotor cortex processes 3D shape from disparity. Neuroimage 47, 262-272. doi: 10.1016/j.neuroimage.2009.04.043

Kayaert, G., Biederman, I., Op de Beeck, H. P., and Vogels, R. (2005). Tuning for shape dimensions in macaque inferior temporal cortex. Eur. J. Neurosci. 22, 212-224. doi: 10.1111/j.1460-9568.2005.04202.x

Kobatake, E., Wang, G., and Tanaka, K. (1998). Effects of shape discrimination training on the selectivity of inferotemporla cells in adult monkeys. J. Neurophysiol. 80, 324-330.

Koida, K., and Komatsu, H. (2007). The effects of task demands on the responses of color-selective neurons in the inferior temporal cortex. Nat. Neurosci. 10, 108-116. doi: 10.1038/nn1823

Kolster, H., Janssen, T., Orban, G. A., and Vanduffel, W. (2014). The retinotopic organization of macaque ocipitotemporal cortex anterior to V4 and caudo-ventral to the MT cluster. J. Neurosci. (in press).

Kolster, H., Mandeville, J. B., Arsenault, J. T., Ekstrom, L. B., Wald, L. L., and Vanduffel. W. (2009). Visual field map clusters in macaque extrastriate visual cortex. J. Neurosci. 29, 7031-7039. doi: 10.1523/JNEUROSCI.051809.2009

Kolster, H., Peeters, R., and Orban, G. A. (2010). The retinotopic organization of the human middle temporal area MT/V5 and its cortical neighbors. J. Neurosci. 30, 9801-9820. doi: 10.1523/JNEUROSCI.2069-10.2010

Kolster, H., Peeters, R., and Orban, G. A. (2011). Ten retinotopically organized areas in the human parietal cortex. Soc. Neurosci. Abstr. 851:10.

Kornblith, S., Cheng, X., Ohayon, S., and Tsao, D. Y. (2013). A network for scene processing in the macaque temporal lobe. Neuron 79, 766-781. doi: 10.1016/j.neuron.2013.06.015

Köteles, K., De Mazière, P. A., Van Hulle, M., Orban, G. A., and Vogels, R. (2008). Coding of images of materials by macaque inferior temporal cortical neurons. Eur. J. Neurosci. 27, 466-482. doi: 10.1111/j.1460-9568.2007.06008.x

Kovacs, G. Y., Vogels, R., and Orban, G. A. (1995). Cortical correlate of pattern backward masking. Proc. Nat. Acad. Sci. U.S.A. 92, 5587-5592. doi: 10.1073/pnas.92.12.5587

Kravitz, D. J., Saleem, K. S., Baker, C. I., Ungerleider, L. G., and Mishkin, M. (2013). The ventral visual pathway: an expanded neural framework for the processing of object quality. Trends Cogn. Sci. 17, 26-49. doi: 10.1016/j.tics.2012.10.011
Ku, S. P., Tolias, A. S., Logothetis, N. K., and Goense, J. (2011). fMRI of the face-processing network in the ventral temporal lobe of awake and anesthetized macaques. Neuron 70, 352-362. doi: 10.1016/j.neuron.2011.02.048

Lafer-Sousa, R., and Conway, B. R. (2013). Parallel, multi-stage processing of colors, faces and shapes in macaque inferior temporal cortex. Nat. Neurosci. 16, 18701878. doi: $10.1038 / \mathrm{nn} .3555$

Larsson, J., and Heeger, D. J. (2006). Two retinotopic visual areas in human lateral occipital cortex. J. Neurosci. 26, 13128-13142. doi: 10.1523/JNEUROSCI.165706.2006

Levy, I., Hasson, U., Avidan, G., Hendler, T., and Malach, R. (2001). Centerperiphery organization of human object areas. Nat. Neurosci. 4, 533-539.

Logothetis, N., Peled, S., and Pauls, J. (1998). Development and application of fMRI for visual studies in monkeys. Soc. Neurosci. Abstr. U.S.A., 28.

Logothetis, N. K., and Sheinberg, D. L. (1996). Visual object recognition. Ann. Rev. Neurosci. 19, 577-621. doi: 10.1146/annurev.ne.19.030196.003045

Mars, R. B., Sallet, J., Neubert, F. X., and Rushworth, M. F. (2013). Connectivity profiles reveal the relationship between brain areas for social cognition in human and monkey temporoparietal cortex. Proc. Natl. Acad. Sci. U.S.A. 110, 1080610811. doi: 10.1073/pnas.1302956110

Miyamoto, K., Adachi, Y., Osada, T., Watanabe, T., Kimura, H. M., Setsuie, R., et al. (2014). Dissociable memory traces within the macaque medial temporal lobe predict subsequent recognition performance. J. Neurosci. 34, 1988-1197. doi: 10.1523/JNEUROSCI.4048-13.2014

Moeller, S., Freiwald, W. A., and Tsao, D. Y. (2008). Patches with links: a unified system for processing faces in the macaque temporal lobe. Science 320, 1355-1359. doi: $10.1126 /$ science. 1157436

Nasr, S., Liu, N., Devaney, K. J., Yue, X., Rajimehr, R., Ungerleider, L. G., et al. (2011). Scene-selective cortical regions in human and nonhuman primates. J. Neurosci. 31, 13771-13785. doi: 10.1523/JNEUROSCI.2792-11.2011

Nassi, J. J., and Callaway, E. M. (2009). Parallel processing strategies of the primate visual system. Nat. Rev. Neurosci. 10, 360-372. doi: 10.1038/nrn2619

Naya, Y., Yoshida, M., and Miyashita, Y. (2003a). Forward processing of long-term associative memory in monkey inferotemporal cortex. J. Neurosci. 23, 2861-2871.

Naya, Y., Yoshida, M., Takeda, M., Fujimichi, R., and Miyashita, Y. (2003b). Delayperiod activities in two subdivisions of monkey inferotemporal cortex during pair association memory task. Eur. J. Neurosci. 18, 2915-2918. doi: 10.1111/j.14609568.2003.03020.x

Nelissen, K., Borra, E., Gerbella, M., Rozzi, S., Luppino, G., Vanduffel, W., et al. (2011). Action observation circuits in the macaque monkey cortex. J. Neurosci. 31, 3743-3756. doi: 10.1523/JNEUROSCI.4803-10.2011

Nelissen, K., Joly, O., Durand, J. B., Todd, J. T., Vanduffel, W., and Orban, G. A. (2009). The extraction of depth structure from shading and texture in the macaque brain. PLoS ONE 4:e8306. doi: 10.1371/journal.pone.0008306

Ohayon, S., Freiwald, W. A., and Tsao, D. Y. (2012). What makes a cell face selective? The importance of contrast. Neuron 74, 567-581. doi: 10.1016/j.neuron.2012.03.024

Okazawa, G., Goda, N., and Komatsu, H. (2012). Selective responses to specular surfaces in the macaque visual cortex revealed by fMRI. Neuroimage 63, 13211333. doi: 10.1016/j.neuroimage.2012.07.052

Olson, I. R., Plotzker, A., and Ezzyat, Y. (2007). The enigmatic temporal pole: a review of findings on social and emotional processing. Brain 130, 1718-1731. doi: 10.1093/brain/awm052

Op De Beeck, H., and Vogels, R. (2000). Spatial sensitivity of macaque inferior temporal neurons. J. Comp. Neurol. 426, 505-518. doi: 10.1002/10969861(20001030)426:4<505::AID-CNE1>3.0.CO;2-M

Orban, G. A. (2008). Higher order visual processing in macaque extrastriate cortex. Physiol. Rev. 88, 59-89. doi: 10.1152/physrev.00008.2007

Orban, G. A. (2011). The extraction of 3D shape in the visual system of human and nonhuman primates. Annu. Rev. Neurosci. 34, 361-388. doi: 10.1146/annurevneuro-061010-113819

Orban, G. A., and Jastorff, J. (2014). "Functional mapping of motion regions in human and non-human primates," in The New Visual Neurosciences, eds J. S. Werner and L. M. Chalupa (Cambridge: MIT press), 777-791.

Orban, G. A., and Vogels, R. (1998). The neuronal machinery involved in successive discrimination. Prog. Neurobiol. 55, 117-147. doi: 10.1016/S03010082(98)00010-0

Pasupathy, A., and Connor, C. E. (2001). Shape representation in area V4: position-specific tuning for boundary conformation. J. Neurophysiol. 86, 25052519. 
Peuskens, H., Claeys, K. G., Todd, J. T., Norman, J. F., Van Hecke, P., and Orban G. A. (2004). Attention to 3-D shape, 3-D motion, and texture in 3-D structure from motion displays. J. Cogn. Neurosci. 16, 665-682. doi: 10.1162/089892904323057371

Pinsk, M. A., Arcaro, M., Weiner, K. S., Kalkus, J. F., Inati, S. J., Gross, C. G., et al. (2009). Neural representations of faces and body parts in macaque and human cortex: a comparative FMRI study. J. Neurophysiol. 101, 2581-2600. doi: 10.1152/jn.91198.2008

Pitzalis, S., Galletti, C., Huang, R. S., Patria, F., Committeri, G., Galati, G., et al. (2006). Wide-field retinotopy defines human cortical visual area v6. J. Neurosci. 26, 7962-7973. doi: 10.1523/JNEUROSCI.0178-06.2006

Polosecki, P., Moeller, S., Schweers, N., Romanski, L. M., Tsao, D. Y., and Freiwald, W. A. (2013). Faces in motion: selectivity of macaque and human face processing areas for dynamic stimuli. J. Neurosci. 33, 11768-11773. doi: 10.1523/JNEUROSCI.5402-11.2013

Popivanov, I. D., Jastorff, J., Vanduffel, W., and Vogels, R. (2012). Stimulus representations in body-selective regions of the macaque cortex assessed with event-related fMRI. Neuroimage 63, 723-741. doi: 10.1016/j.neuroimage.2012.07.013

Quiroga, R. Q. (2012). Concept cells: the building blocks of declarative memory functions. Nat. Rev. Neurosci. 13, 587-597. doi: 10.1038/nrn3251

Quiroga, R. Q., Reddy, L., Kreiman, G., Koch, C., and Fried, I. (2005). Invariant visual representation by single neurons in the human brain. Nature 435, 1102 1107. doi: 10.1038 /nature 03687

Saleem, K. S., Kondo, H., and Price, J. L. (2008). Complementary circuits connecting the orbital and medial prefrontal networks with the temporal, insular, and opercular cortex in the macaque monkey. J. Comp. Neurol. 506, 659-693. doi: $10.1002 / \mathrm{cne} .21577$

Saleem, K. S., Price, J. L., and Hashikawa, T. (2007). Cytoarchitectonic and chemoarchitectonic subdivisions of the perirhinal and parahippocampal cortices in macaque monkeys. J. Comp. Neurol. 500, 973-1006. doi: 10.1002/cne. 21141

Sallet, J., Mars, R. B., Noonan, M. P., Andersson, J. L., O’Reilly, J. X., Jbabdi, S., et al. (2011). Social network size affects neural circuits in macaques. Science 334, 697-700. doi: 10.1126/science.1210027

Sato, T., Uchida, G., Lescroart, M. D., Kitazono, J., Okada, M., and Tanifuji, M. (2013). Object representation in inferior temporal cortex is organized hierarchically in a mosaic-like structure. J. Neurosci. 33, 16642-16656. doi: 10.1523/JNEUROSCI.5557-12.2013

Sawamura, H., Georgieva, S., Vogels, R., Vanduffel, W., and Orban, G. A. (2005). Using functional magnetic resonance imaging to assess adaptation and size invariance of shape processing by humans and monkeys. J. Neurosci. 25, 4294-4306. doi: 10.1523/JNEUROSCI.0377-05.2005

Saxe, R., Carey, S., and Kanwisher, N. (2004). Understanding other minds: linking developmental psychology and functional neuroimaging. Annu. Rev. Psychol. 55 87-124. doi: 10.1146/annurev.psych.55.090902.142044

Sereno, M. E., Trinath, T., Augath, M., and Logothetis, N. K. (2002). Threedimensional shape representation in monkey cortex. Neuron 33, 635-652. doi: 10.1016/S0896-6273(02)00598-6

Sigala, N., and Logothetis, N. K. (2002). Visual categorization shapes feature selectivity in the primate temporal cortex. Nature 415, 318-320. doi: $10.1038 / 415318 \mathrm{a}$

Silver, M. A., and Kastner, S. (2009). Topographic maps in human frontal and parietal cortex. Trends Cogn. Sci. 13, 488-495. doi: 10.1016/j.tics.2009.08.005

Silver, M., A., Ress, D., and Heeger, D. J. (2005). Topographic maps of visual spatial attention in human parietal cortex. J. Neurophysiol. 94, 1358-1371. doi: 10.1152/jn.01316.2004

Singer, J. M., and Sheinberg, D. L. (2010). Temporal cortex neurons encode articulated actions as slow sequences of integrated poses. J. Neurosci. 30, 3133-3145. doi: 10.1523/JNEUROSCI.3211-09.2010

Srihasam, K., Mandeville, J. B., Morocz, I. A., Sullivan, K. J., and Livingstone, M. S. (2012). Behavioral and anatomical consequences of early versus late symbo training in macaques. Neuron 73, 608-619. doi: 10.1016/j.neuron.2011.12.022

Stefanacci, L., Reber, P., Costanza, J., Wong, E., Buxton, R., Zola, S., et al. (1998) fMRI of monkey visual cortex. Neuron 20, 1051-1057. doi: 10.1016/S08966273(00)80485-7

Takechi, H., Onoe, H., Shizuno, H., Yoshikawa, E., Sadato, N., Tsukuda, H., et al (1997). Mapping of cortica area sinvolved in color vision in non-human primates. Neurosci. Lett. 230, 17-20. doi: 10.1016/S0304-3940(97)00461-8
Takeda, M., Naya, Y., Fujimichi, R., Takeuchi, D., and Miyashita, Y. (2005). Active maintenance of associative mnemonic signal in monkey inferior temporal cortex. Neuron 48, 839-84 doi: 10.1016/j.neuron.2005.09.028

Takeuchi, D., Hirabayashi, T., Tamura, K., and Miyashita, Y. (2011). Reversal of interlaminar signal between sensory and memory processing in monkey temporal cortex. Science 331, 1443-1447. doi: 10.1126/science.1199967

Tanaka, K. (1996). Inferotemporal cortex, object vision. Annu. Rev. Neurosci. 19, 109-139. doi: 10.1146/annurev.ne.19.030196.000545

Tanaka, K., Saito, H., Fukuda, Y., and Moriya, M. (1991). Coding visual images of objects in infero-temporal cortex of the macaque monkey. J. Neurophysiol. 66, 170-189.

Tootell, R. B., Mendola, J. D., Hadjikhani, N. K., Ledden, P. J., Liu, A. K., Reppas, J. B., et al. (1997). Functional analysis of V3A and related areas in human visual cortex. J. Neurosci. 17, 7060-7078.

Tsao, D. Y., Freiwald, W. A., Knutsen, T. A., Mandeville, J. B., and Tootell, R. B. (2003). Faces and objects in macaque cerebral cortex. Nat. Neurosci. 6, 989-995. doi: $10.1038 / \mathrm{nn} 1111$

Tsao, D. Y., Freiwald, W. A., Tootell, R. B., and Livingstone, M. S. (2006). A cortical region consisting entirely of face-selective cells. Science 311, 670-674. doi: $10.1126 /$ science. 1119983

Ungerleider, L. G., Galkin, T. W., Desimone, R., and Gattass, R. (2008). Cortical connections of area V4 in the macaque. Cereb. Cortex 18, 477-499. doi: 10.1093/cercor/bhm061

Vandenberghe, R., Price, C., Wise, R., Josephs, O., and Frackowiak, R. S. (1996). Functional anatomy of a common semantic system for words and pictures. Nature 383, 254-256. doi: 10.1038/383254a0

Vanduffel, W., Beatse, E., Sunaert, S., Van Hecke, P., Tootell, R. B. H., and Orban, G. A. (1998). Functional magnetic resonance imaging in an awake rhesus monkey. Soc. Neurosci. Abstr. 24, 11.

Vanduffel, W., Fize, D., Mandeville, J. B., Nelissen, K., Van Hecke, P., Rosen, B. R., et al. (2001). Visual motion processing investigated using contrast agent-enhanced fMRI in awake behaving monkeys. Neuron 32, 565-577. doi: 10.1016/S0896-6273(01)00502-5

Vangeneugden, J., Pollick, F., and Vogels, R. (2009). Functional differentiation of macaque visual temporal cortical neurons using a parametric action space. Cereb. Cortex 19, 593-611. doi: 10.1093/cercor/bhn109

Vogels, R., and Orban, G. A. (1994). Does practice in orientation discrimination lead to changes in the response properties of macaque inferior temporal neurons? Eur. J. Neurosci. 6, 1680-1694. doi: 10.1111/j.1460-9568.1994.tb00560.x

Wandell, B. A., Dumoulin, S. O., and Brewer, A. A. (2007). Visual field maps in human cortex. Neuron 56, 366-383. doi: 10.1016/j.neuron.2007.10.012

Yamane, Y., Carlson, E. T., Bowman, K. C., Wang, Z., and Connor, C. E. (2008). A neural code for three-dimensional object shape in macaque inferotemporal cortex. Nat. Neurosci. 11, 1352-1360. doi: 10.1038/nn.2202

Zeki, S. M. (1978). Functional specialisation in the visual cortex of the rhesus monkey. Nature 274, 423-428. doi: 10.1038/274423a0

Zeki, S., and Marini, L. (1998). Three cortical stages of colour processing in the human brain. Brain 121, 1669-1685. doi: 10.1093/brain/121.9.1669

Zhang, N. R., and von der Heydt, R. (2010). Analysis of the context integration mechanisms underlying figure-ground organization in the visual cortex. J. Neurosci. 30, 6482-6496. doi: 10.1523/JNEUROSCI.5168-09.2010

Conflict of Interest Statement: The authors declare that the research was conducted in the absence of any commercial or financial relationships that could be construed as a potential conflict of interest.

Received: 19 February 2014; accepted: 16 June 2014; published online: 02 July 2014. Citation: Orban GA, Zhu Q and Vanduffel W (2014) The transition in the ventral stream from feature to real-world entity representations. Front. Psychol. 5:695. doi: 10.3389/fpsyg.2014.00695

This article was submitted to Perception Science, a section of the journal Frontiers in Psychology.

Copyright (c) 2014 Orban, Zhu and Vanduffel. This is an open-access article distributed under the terms of the Creative Commons Attribution License (CC BY). The use, distribution or reproduction in other forums is permitted, provided the original author(s) or licensor are credited and that the original publication in this journal is cited, in accordance with accepted academic practice. No use, distribution or reproduction is permitted which does not comply with these terms. 